at $42^{\circ} \mathrm{C}$ in a microaerophilic atmosphere. Plates were read at 24 and 48 hours, and positive results were reported on the basis of colonial appearance and microscopic morphology. Both laboratories participate in the UK NEQAS scheme for faecal specimens.

The study period comprised the whole of 1988 and 1989, and 3287 specimens were processed in parallel. The results are shown in the table. The isolation rate of Campylobacter was significantly better $(p \leqslant 0.001)$ from specimens processed promptly.

There are several variables here, but our culture method works satisfactorily for fresh specimens in both laboratories, and the most obvious reason for the difference is the delay in culturing specimens at the large laboratory.

Storage and transport of Campylobacter jejuni has been studied by Monfort et al, ${ }^{1}$ who found that the organism survives in animal faeces for at least three hours at $4^{\circ} \mathrm{C}$ but for less than two hours at $25^{\circ} \mathrm{C}$; holding atmosphere had no effect on isolation rates. Our experience is that Campylobacter sp survive for over a week at $4^{\circ} \mathrm{C}$ in Stuart's transport medium. The organism may be killed by toxic metabolites, and transport at ambient temperature may be the real problem.

Thus centralisation would result in a significant false negative rate, whereas culture at the small laboratory would yield more positive results a day earlier. Other alternatives could include using transport medium (which would entail handling the specimen almost as much as direct culture) or enrichment (which would add another day to the delay already experienced); both would add to the cost.

Our observations also have implications for provision of services or storage of specimens at weekends and Bank holidays.

CJ BUTT Yeovil District Hospital, Yeovil, Somer MCJ WALE Department of Microbiology, City Hospital, Nottingham NG5 $1 P B$ of sample holding time, temperature, and atmosphere on the isolation of Campylobacter ejuni from dogs. $J$ Clin Microbio 1989;27:1419-20.

\section{Flow cytometric DNA in ectopic pregnancy}

The estimated incidence of ectopic pregnancy varies from 1 in 84 to 1 in 230 intrauterine pregnancies. ${ }^{1}$ There are several maternal factors associated with ectopic implantation, such as anatomical changes in the tube involved. These factors are probably more important than the less well documented factors inherent in the embryo. According to various reports, the range of abnormal embryogenesis associated with ectopic pregnancy varies from $0 \%$ to $63.6 \% .^{2}$ It is difficult to ascertain from the information available whether this abnormal embryogenesis is due to increased incidence of chromosomal aberrations. The actual incidence of chromosomal anomalies in ectopic pregnancy remains largely obscure as specimens are rarely available for karyotype analysis, or karyotype analysis cannot be carried out for technical reasons.

Flow cytometric DNA analysis is rapid and relatively simple but can only show relatively gross DNA dearrangements in cell suspensions prepared from paraffin wax embedded solid tissues. The DNA contents of individual chromosomes ranges from 4.3\% (chromosome No 1) to $0.8 \%$ (chromosome No 21) when expressed as a percentage of total autosomal DNA. ${ }^{3}$ Thus in ideal conditions with fresh tissues a DNA difference of at least $8-10 \%$ would be required to detect two mixed cell populations by DNA flow cytometry. ${ }^{4}$ This means that DNA flow cytometry would only show DNA aneuploidy if a substantial amount of the analysed cells lose or acquire several chromosomes.

We performed flow cytometric DNA analysis of 42 randomly selected cases of conceptual mass removed from the fallopian tubes. The histological material consisted of varying amounts of trophoblastic tissue with or without gestational sac and embryo, and of maternal blood. Single cell suspensions for flow cytometric analysis were prepared as described by Schutte et $a l^{5}$ with a few modifications. Quantitative DNA analysis was performed using an EPICS-C (Coulter Electronics, Hialeah, Florida, USA) flow cytometer. For each DNA histogram up to 10000 nuclei were scanned. The mean coefficient of variation (CV) of $8.2 \%$ was obtained (range 4.6-12.0). The DNA index (DI) for each case was calculated. The peak having the lowest channel number was considered to represent diploid cells and to have a DI of 1 0 . DNA hypoploid populations might not have been identified due to the definition of the $G_{0} G_{1}$ peak used and due to the use of dewaxed samples characterised by excessive cell debris. The DNA Index (DI) of a given aneuploid peak was calculated by dividing its channel number by that of the diploid cells in the same sample. Tetraploidy was considered when the G2/M peak consisted of more than $15 \%$ of the total cell count.

Tubal implantation was associated with aneuploidy in $14(33 \%)$ cases. The DNA indices of the aneuploid peaks ranged from $1 \cdot 14$ to $2 \cdot 17$. Eight $(19 \%)$ cases showed karyotype changes in the near diploid region. Six cases were tetraploid. Triploid cases (DI 1.4-1.6) were not recorded.

The results of this study support earlier reported overall incidences of cytogenetic anomalies in ectopic pregnancy. ${ }^{67}$ It is quite possible that chromosomal aberrations contribute significantly to the incidence of ectopic gestation, especially when the tubes seem to be grossly normal or ectopic pregnancy is recurrent. The prevalence of DNA tetraploidy was high. No triploidy-that is, DNA aneuploidy associated with partial mole morphology - was detected.

Flow cytometric DNA analysis can be of positive use in the evaluation of ectopic pregnancies. The advantage of flow cytometric analysis over conventional cytogenetics is the simplicity of the method. At the moment DNA flow cytometry is not as sensitive as cytogenetic techniques for detecting small chromosomal changes. Nevertheless, it can provide interesting but approximate information on the DNA content of the cells under study and thus on the role of karyotype abnormalities in ectopic pregnancy.
R AINE

R KARIKOSKI-LEO Department of Pathology $P$ K HEINONEN Department of Obstetrics and Gynecology,
University Central Hospital of Tampere Finland

1 Elias S, LeBeau M, Simpson JL, Martin AO. Chromosome analysis of ectopic human conceptuses. Am J Obstet Gynecol 1981;141: 698-703.

2 Sopelak VM, Bates GW. Role of transmigration and abnormal embryogenesis in ectopic pregand abnormal embryogenesis in ectopic preg

3 Mendelsohn ML, Mayall BH, Bogart E, Moor II DH, Perry BH. DNA content and DNAbased centromeric index of the 24 human chromosomes. Science 1973;179:1126-9.

4 Vindelov LL, Christensen IJ, Jensen G, Nissen NI. Limits of detection of nuclear DNA abnormalities by flow cytometric DNA analysis. Results obtained by a set of methods for sample storage, staining and internal standardization. Cytometry 1983;3:332-9.

5 Schutte B, Reynders MMJ, Bosman FT, Blijham GH. Flow cytometric determination of DNA-ploidy level in nuclei isolated from of paraffin

6 Poland BJ, Dill FJ, Styblo C. Embryonic development in ectopic human pregnancy. Teratology 1976;14:315-22.

7 Stratford BF. Abnormalities of early human development. Am J Obstet Gynecol 1970; 107:1223-32.

MATTERS ARISING

Monocyte esterase deficiency: familial or environmental?

A recent report by Markey et al presents some intriguing results concerning monocyte esterase deficiency and malignant neoplasia. ${ }^{\prime}$ They found a significant deficiency in $0.8 \%$ of blood donors, in $3.9 \%$ of patients with malignancies, and in $1.7 \%$ of patients with non-malignant diseases. Their method for measuring monocyte esterase deficiency was the Technicon Hemalog D automated white cell differential counter. This system uses the presence of esterase in monocytes as a means of distinguishing monocytes from neutrophils. The Hemalog $\mathrm{D}$ has been in use world wide since 1974, but has mostly now been phased out by the more recent Technicon $\mathrm{H}$ 6000 and $\mathrm{H}-1$ systems. These current systems do not use esterase staining, and thus make the study performed by Markey et al difficult to repeat. Two other semiautomated methods for the measurement of monocyte esterase offer possible substitutes. Markovic et al report an image processing system which measures the esterase of blood cell smear preparations. ${ }^{2} \mathrm{My}$ laboratory recently reported an adaptation of the Hemalog D chemistry for use on the current Technicon $\mathrm{H}-1$ system. ${ }^{3}$ Our method, like that in use with the Hemalog D, stains cells in suspension using a whole blood sample.

The observations by Markey et al, while intriguing, do require further experimentation to test their hypothesis that "severe diminution in monocyte esterase activity, which occurs in an inheritable form and is relatively common, may at least in a minor 